Les sources de l'Histoire des Mines : Nouveaux outils, Nouvelles approches

\title{
Les archives des mines : un produit administratif, une source pour l'histoire
}

The mines archives : the product of an administration and a source for history

\section{Bruno Belhoste}

\section{(2) OpenEdition}

\section{Journals}

Édition électronique

URL : http://journals.openedition.org/dht/746

DOI : $10.4000 /$ dht.746

ISSN : $1775-4194$

Éditeur :

Centre d'histoire des techniques et de l'environnement du Cnam (CDHTE-Cnam), Société des élèves du CDHTE-Cnam

\section{Édition imprimée}

Date de publication : 1 décembre 2008

Pagination : 4-8

ISBN : 978-2-95-30779-2-6

ISSN : 0417-8726

Référence électronique

Bruno Belhoste, "Les archives des mines : un produit administratif, une source pour l'histoire », Documents pour l'histoire des techniques [En ligne], 16 | $2^{\mathrm{e}}$ semestre 2008, mis en ligne le 06 octobre 2010, consulté le 08 septembre 2020. URL : http://journals.openedition.org/dht/746 ; DOI : https:// doi.org/10.4000/dht.746 


\title{
Les archives des mines: un produit administratif, une source pour l'histoire
}

\author{
Bruno Belhoste \\ EA $127 / \mathrm{CH} 2 \mathrm{ST}$ \\ Université Paris 1 Panthéon Sorbonne
}

\begin{abstract}
RÉSUMÉ
L'histoire ne peut se faire sans archives. Cette vérité toute simple est source elle-même de questionnements pour l'historien : qui a fabriqué les archives et dans quels buts? Comment se sont-elles conservées et pourquoi ? Pour répondre à ces questions, I'historien a besoin de la collaboration des experts que sont les archivistes et les conservateurs. L'histoire des mines en fournit une bonne illustration. L'immense mine documentaire à la disposition des historiens y est le résultat d'une intense activité administrative. Son exploitation comme source exige d'en comprendre d'abord les modes de production, d'usage et de conservation. Si un tel travail est la condition d'un usage pertinent de la documentation, il ouvre aussi sur un vaste champ d'études : celui des cultures de travail et des pratiques d'écriture d'une administration technique.
\end{abstract}

Résumés et mots clés en anglais sont regroupés en fin de volume, accompagnés des mots clés français

C ette introduction sera modeste. L'important, bien sûr, ce sont les communications ciblées qui suivent, sur les fonds d'archives et les outils pour les exploiter. Je me contenterai ici de fournir quelques éléments pour nourrir la réflexion, sans prétendre imposer un cadre à ce recueil.

Il est à peine besoin de dire l'importance historique de l'activité minière en France. Les mines ont constitué dans de nombreuses régions une activité essentielle, surtout entre 1820 et 1960, pendant la grande période de l'industrie. Des hommes en grand nombre, des communautés, des institutions et l'État lui-même ont été concernés et engagés dans cette activité. Aujourd'hui, l'activité minière a quasiment disparu du territoire national. La dernière mine de fer a fermé en 1997, la dernière mine de charbon en avril 2004. En Lorraine, les mines de fer viennent d'être ennoyées. À la fin de l'année 2007, la société Charbonnages de France a mis la clé sous la porte, pour disparaître définitivement. C'est la fin d'une histoire et la fin d'un monde. En France, les mines sont maintenant, pour l'essentiel, une affaire de mémoire : on voit fleurir les Musées de la Mine, paraître les témoignages et les évocations, s'empiler une documentation archivée devenue inutile.

L'historien de son côté s'intéresse bien sûr depuis longtemps à l'histoire des mines. II en connaît l'importance pour l'histoire industrielle, économique, sociale et culturelle de notre pays. Or pour faire de l'histoire, I'historien a besoin de sources. En disposer, d'accessibles et de bien classées, est donc pour lui essentiel. L'historien positiviste d'autrefois pensait que son travail consistait à dégager les faits positifs et incontestables à partir de la documentation léguée et conservée, en procédant à un travail rigoureux de critique, de vérification et de croisement des sources. L'historien d'aujourd'hui est plus ambitieux, ou plus prétentieux. ॥ aimerait en effet construire ses sources, voire les inventer. Pour cela, pense-t-il, il est possible de faire parler l'archive, de lui faire dire ce qu'elle ne voulait pas dire, de faire surgir, en somme, le non-dit du dit. L'historien serait ainsi un peu comme le physicien dans son laboratoire, qui ne se contente pas d'observer la nature, mais qui la fait parler en inventant des dispositifs expérimentaux toujours plus sophistiqués. 
Cette inventivité de l'historien de la fin du XXe siècle et du début de notre siècle a produit des résultats remarquables, renouvelant I'historiographie et découvrant des pans d'histoire qui auraient semblé inaccessibles à l'historien d'antan. Mais il en est résulté une certaine tendance à oublier ce qui fait, malgré tout, le fondement du travail de l'historien, la matière de ses sources, c'est-à-dire l'archive elle-même. Or, en ne s'intéressant pas à l'archive, à sa production et à son invention, car il y a une invention de l'archive comme il y a une invention des sources, l'historien risquerait un véritable appauvrissement de l'histoire, faute d'un renouvellement de son carburant. C'est pourquoi le temps est venu pour lui, je crois, de revenir à l'archive, à la documentation primaire, pour comprendre comment elle s'invente, d'où elle vient, comment elle se forme, comment elle se dépose, se conserve et finalement se transforme. Dans ce travail à reprendre, l'historien n'est pas seul bien sûr. Il a avec lui, beaucoup plus conscient et compétent que lui, le professionnel des archives, l'archiviste-paléographe, le conservateur. Celui-ci doit être plus, pour l'historien, qu'un simple auxiliaire, qu'un technicien trop souvent invisible, il doit être, à égalité avec lui, un collaborateur et un soutien. Car l'historien a tendance à oublier que l'archive n'est pas une donnée naturelle, évidente, qu'elle n'est pas, si je puis dire, une mine à ciel ouvert, dans les magasins des archives. L'archiviste-paléographe, lui, en est pleinement conscient. C'est son métier, c'est son pain quotidien. Pour le domaine qui nous intéresse, l'entreprise engagée depuis 2004 par Bruno Delmas, Denise Ogilvie, Dominique Margairaz sur la construction, la conservation et la circulation des corpus documentaires relatifs aux savoirs administratifs, qui réunit historiens et professionnels des archives, fournit de ce point de vue un modèle exemplaire '.

En amont de l'archive sur l'activité minière il y a donc une longue chaîne administrative, qui va des producteurs d'archives à ceux qui les trient et les conservent pour la postérité. Considérons d'abord l'étape initiale. C'est dans les bureaux et non au fond de la mine que la documentation se fabrique et s'empile. Nous

1 Sur ce projet, voir Bruno Delmas, « Archéologie des savoirs administratifs. Construction, conservation et circulation des corpus (XVIIIe-XIXe siècles) "1, dans Karine Chemla éd., Action concertée Histoire des savoirs, 2003-2007. Recueil de synthèses, Paris, CNRS, 2007, pp. 228-234. Voir aussi un numéro thématique de la Bibliothèque de l'École des chartes à paraître en 2008 sur le thème « De l'Ancien Régime à l'Empire : mutations de l'État et avatars d'archives $॥$. sommes ainsi conduits naturellement à nous intéresser au mode de production de l'écrit administratif dans l'univers minier. II faut connaître et décrire les lieux institutionnels et géographiques de l'activité papier : qu'elle soit locale ou nationale, au niveau de l'entreprise minière, de l'arrondissement administratif ou de I'administration centrale. || faut connaître et décrire les modalités de circulation de cette documentation, ses montées et descentes dans la structure bureaucratique, ses lieux d'arrêt et de stockage, ses lieux de reformulation et de synthèse. Il faut connaître et décrire les pratiques du travail administratif, son organisation, ses normes et ses codes : comment rédige-t-on un rapport ou une statistique? À qui sont-ils destinés et pour quels usages ? II faut enfin connaître et décrire les acteurs qui interviennent dans la production de cette chaîne de papier, les auteurs, les lecteurs et les décideurs

Voilà qui nous amène aux cultures et aux savoirs qui sont intégrés dans la routine administrative. Ces producteurs de papiers sont des hommes formés, pour ne pas dire formatés. Beaucoup, les plus gradés, sont passés par des établissements où ils ont appris des sciences et des savoir-faire de haut niveau, qui leur servent de référents : à l'École polytechnique, à l'École des mines de Paris et, plus bas dans la hiérarchie, dans les Écoles de mineurs en province. Mais il faut considérer également la culture technique acquise sur le terrain, la culture politique acquise dans les assemblées et les salons, la culture juridique acquise au contact des hommes de loi, qui ont aussi à traiter, et à tous les niveaux, des questions de propriété et d'exploitation minière. Ces cultures ne sont pas seulement des cultures de sociabilité, ce sont des cultures de l'écrit et des cultures de travail. Les gisements d'archives présentés dans les communications qui suivent sont justement le produit de ce travail. On y verra la description de certains fonds et l'indication de leurs origines et de leur mode de formation et d'accumulation. Je me contenterai ici d'évoquer quelquesuns des producteurs d'archives minières. Ce faisant, c'est le fonctionnement de l'appareil bureaucratique qui les a fabriquées par son activité que je serai ainsi conduit à examiner.

Je rappelle que c'est seulement en 1781 que Necker établit une modeste administration des mines et des minières, composée de 4 inspecteurs, administration que l'on peut considérer comme l'embryon de l'administration des mines d'aujourd'hui ${ }^{2}$. Pendant

2 Sur l'origine du corps des mines et de l'École des mines au $\mathrm{XVIII}{ }^{\mathrm{e}}$ siècle, le travail de référence reste Arthur Birembaut, " L'enseignement de la minéralogie et des techniques mi- 


\section{Les sources de l'histoire des mines}

longtemps, faute d'une politique minière, l'État royal se contentait de commissionner à l'occasion des ingénieurs de l'industrie privée pour enquêter sur les mines du royaume. Nous en savons un peu plus sur cette période grâce à Christiane Demeulenaere et son étude sur l'enquête du Régent, présenté dans ce numéro. II a fallu attendre l'instauration en 1744 d'un régime de concession pour l'exploitation des mines de charbon pour que le Contrôle général se préoccupe enfin de ces questions. C'est alors que l'État forme ses premiers inspecteurs, dont le fameux Antoine Gabriel Jars, à l'École des ponts et chaussées nouvellement créée. Mais le Contrôle général renonce finalement, pour des raisons d'économie, à constituer un service permanent d'inspection. Deux ans, donc, après la création de la nouvelle administration, en 1781, une École des mines est installée à la Monnaie, autour de la chaire publique de minéralogie docimastique créée en 1778 pour le chimiste Balthazar Georges Sage de l'Académie royale des sciences. Mais en 1789, le département des mines reste une institution à l'identité fragile, dont le personnel -2 commissaires, 6 inspecteurs, 2 sous inspecteurs, 6 ingénieurs et 6 élèves des mines - d'origines diverses, manque encore singulièrement d'homogénéité. Après un passage difficile, la nouvelle administration réussit à traverser la Révolution sans disparaître et elle voit finalement son statut fixé pour longtemps avec la loi du 21 avril 1810 et le décret du 10 novembre 1810, comme l'explique Lionel Latty dans sa communication.

L'administration des Mines dépend du ministère de I'Intérieur jusqu'au début des années 1830, puis du Ministère des travaux publics et ses différents avatars ${ }^{3}$. Sa gestion, au ministère, est étroitement associée à celle des Ponts et chaussées. Ses ingénieurs, organisés en corps (il y en a 150 environ au milieu du XIXe siècle), ont d'ailleurs en commun avec ceux des ponts et chaussées de sortir tous de l'École polytechnique, et parmi les premiers classés. Pour aider le ministre et traiter les dossiers au niveau central, il existe un Conseil général des mines. Le territoire national est divisé en arrondissements miniers, disposant chacun d'une petite structure administrative, composée d'ingénieurs, de conducteurs et de gardes-mine. Le service assuré par l'administration des mines et le corps des mines est un service technique, comme celui des ponts et chaussées. Son action

nières 11, dans René Taton éd., Enseignement et diffusion des sciences au XVIII siècle, Paris, Hermann, 1964, pp. 365-41

3 Voir, sur ce qui suit, André Thépot, Les ingénieurs des mines du XIXe siècle. Histoire d'un corps technique. Tome 1 : 1810-1914, Paris, éditions Eska, 1998. exprime dans le domaine de l'aménagement et de l'industrie l'autorité qu'exerce l'État, avec sa tradition de réglementation et de contrôle dont l'origine remonte loin dans la Monarchie administrative. Selon la loi du 21 avril 1810 et le décret du 10 novembre 1810, la compétence du service des mines s'étend ainsi principalement sur les mines, minières et carrières ainsi que sur les usines sidérurgiques. II donne un avis sur l'autorisation de recherche et d'exploitation minière et sur l'établissement de fourneaux, forges et usines sidérurgiques.

Le Conseil général des mines examine les demandes en concession, les travaux d'art auxquels il conviendra d'assujettir le concessionnaire, comme condition de la concession, les reprises de travaux et l'utilité ou les inconvénients des partages des concessions. Il exerce une surveillance de police des concessions de mines, des carrières et des usines et s'assure de la conservation des édifices et de la sûreté du sol (tournées d'inspection des ingénieurs). ॥ assure la levée de la redevance minière et évalue pour cela le produit des mines, d'où la réalisation de statistiques minières. II peut fournir une expertise sur les mines et l'industrie devant la justice et donner des conseils aux exploitants. Enfin, et c'est fondamental, il suit les questions de sécurité et contribue à l'amélioration des conditions d'exploitation et à la formation des entrepreneurs. En conséquence il développe l'enseignement des mines, administre des écoles et mène d'importantes recherches géologiques et minéralogiques. À ces missions spécifiques s'ajoutent des compétences partagées avec les ingénieurs des ponts et chaussées, comme le contrôle des machines à vapeur ou la surveillance de l'exploitation des chemins de fer qui s'avèreront dans la suite décisives, mais que nous laisserons ici de côté.

À toutes ces missions correspond une importante activité bureaucratique, d'où une grosse production de documents écrits, se présentant sous des formes variées, tels les dossiers, les rapports, les registres, les cartes et les plans, qui sont à la base de nos archives publiques des mines. II s'agit d'abord de dossiers liés à la gestion de la structure et de son personnel. C'est en particulier le cas des dossiers personnels d'ingénieurs, très riches, qui donnent les états de carrière, la notation administrative et la correspondance avec l'administration centrale liée à cette carrière. Ces dossiers, bien connus des historiens, sont dans F/14, aux Archives nationales. II s'agit ensuite des dossiers produits par le travail des services. 
Il faut considérer ceux du service ordinaire et extraordinaire des ingénieurs sur le terrain. L'essentiel est dans la série $S$ des archives départementales. La communication de Denys Barau illustre la richesse de la documentation disponible sur l'exemple particulièrement remarquable du département de la Loire. Y sont conservés les dossiers d'attribution des concessions et tout ce qui concerne le contrôle de l'exploitation par les ingénieurs des mines, en particulier pour ce qui touche à la sécurité. Au niveau de l'administration centrale, la documentation est encore plus considérable. Le registre des procès-verbaux du Conseil général des mines fournit sans doute le meilleur accès à cet ensemble documentaire immense. Tous les dossiers des affaires transmises à Paris par les ingénieurs sont en effet examinés pour avis par le Conseil. La plupart ont donné lieu à la production d'arrêtés et d'ordonnances (après un passage par le Conseil d'État). Quant aux dossiers eux-mêmes, ils ont été dispersés, remembrés et triés dans les bureaux tout au long de leur vie active, avant d'être reversés dans les cadres de classement des archives nationales. Par un travail admirable de bénédictin, Lionel Latty, dont on lira ci-dessous l'article sur la loi de 1810 et l'activité du Conseil général des mines au XIXe siècle, a entrepris de dresser une table analytique détaillée de ces registres, dont la consultation permet dès maintenant de suivre la conduite d'une affaire par l'administration centrale sur la période 1810-1831. Cette table ouvre la voie par la création d'un puissant outil pour reconstituer les dossiers des affaires instruites par le Conseil à partir des pièces aujourd'hui dispersées dans les fonds.

Il faut considérer également, à un autre niveau, l'immense production intellectuelle de l'administration des mines. Les sources imprimées et manuscrites conservées à la Bibliothèque de l'École des mines, présentées ici par Marie-Noëlle Maisonneuve et Francine Masson, sont abondantes. Parmi beaucoup de documents d'archives, mentionnons les rapports et journaux sur les voyages minéralogiques des élèves qui fournissent des informations précises et de première main sur de nombreux sites et usines, en France et à l'étranger. Doit être prise en compte également la production papier liée à la réalisation de la carte géologique de France, à partir de 1825, et à la préparation des statistiques minières et métallurgiques, à partir de 1835, ce qui exige de parcourir les fonds conservés dans la série F/14, mais aussi les papiers des ingénieurs conservés dans de nombreuses bibliothèques, par exemple à l'Institut et, bien sûr, encore une fois, à l'École des mines.
J'ai envisagé jusqu'à présent les produits papier relatifs à l'activité de l'administration des mines. II faudrait bien entendu prendre en compte ceux relatifs à la gestion des compagnies minières elles-mêmes. Je ne m'aventurerai pas sur ce terrain immense et très imparfaitement exploré, mais il est clair qu'il s'agit là encore d'une « mine » très abondante. II suffira de lire, pour ce qui concerne la houille, la communication très éclairante de Pascal Even et Jean-Charles Besson sur la sauvegarde de la mémoire de Charbonnages de France pour mesurer l'incroyable richesse, mais aussi la complexité de ces archives d'entreprise aujourd'hui dispersées en plusieurs lieux. Soulignons seulement ici que, si l'administration des Mines est partie intégrante de l'appareil d'État, dont elle partage les valeurs et les points de vue, elle est aussi directement au contact du monde de l'entreprise et de ses hommes, dont elle n'ignore ni les intérêts ni les pratiques, surtout au plan local ${ }^{4}$.

Après être remonté ainsi en amont jusqu'à la source de l'archive, redescendons maintenant vers l'aval, jusque là où cette archive, conservée, classée et organisée, se transforme à son tour en source pour le travail de l'historien. La lecture des communications rassemblées dans ce recueil nous fait toucher du doigt, sur le cas des mines, l'immense travail requis pour passer de la documentation vivante à l'archive disponible pour le chercheur. Hasards de la conservation, déplacements et déménagements, tris plus ou moins sélectifs et rationnels, classements et inventaires plus ou moins partiels : les articles écrits ici par les professionnels des archives mettent en évidence la difficulté d'une tâche que l'utilisateur final tend à négliger et les facteurs intellectuels, mais aussi financiers, voire politiques et culturels, qui ont contribué et contribuent encore à la constitution des fonds que l'historien utilise et exploite sans avoir toujours suffisamment conscience de ces tribulations.

L'histoire des mines, en tout cas, est assez vaste pour offrir des matériaux à de multiples investigations. Pour une histoire locale, ou régionale, les archives de l'administration minière et celles des compagnies minières fournissent une documentation totale, susceptible elle-même d'être agrégée dans un corpus documentaire plus large. C'est un aspect qu'on ne saurait négliger, mais sur lequel je n'insisterai pas ici. À un autre niveau, le corpus documentaire des mines offre une source privilégiée pour une histoire indus-

4 Voir sur ce thème, Anne-Françoise Garçon, Entre l'État et l'usine. L'École des mines de Saint-Étienne au XIXe siècle, Rennes, PUR, 2004. 


\section{Les sources de l'histoire des mines}

trielle de la France et plus encore pour une histoire de la politique industrielle. Les dossiers sur les accidents miniers apportent des éléments essentiels pour une histoire des populations ouvrières. Les procès-verbaux du Conseil général des mines représentent pour le XIX siècle un observatoire exceptionnel sur l'implantation des activités industrielles lourdes sur le territoire. Bien sûr, il s'agit de sources déjà exploitées par les historiens de l'industrialisation, mais dont la richesse est loin encore d'avoir été épuisée.

Mais l'intérêt de cette mine documentaire ne se limite pas à l'histoire industrielle. Je voudrais insister aussi, puisque c'est mon domaine, sur l'intérêt et l'importance de ce type d'archives pour l'histoire des savoirs en général, celle des savoirs techniques, scientifiques, juridiques et administratifs en particulier. C'est un très vaste champ qui reste encore largement sousétudié. Les travaux de cartographie minéralogique et minière sont considérables. Les techniques de relevé et d'investigation sur le terrain mériteraient d'être décortiquées et analysées. II s'agirait bien sûr de prendre en compte les savoirs de référence, tels qu'on les trouve exposés dans les articles, les cours et les traités, mais aussi les savoirs théorico-pratiques mis en œuvre par les acteurs de terrain dans leur activité professionnelle courante. De manière générale, on aimerait mieux connaître les pratiques d'expertise qui constituent le savoir-faire de l'administrateur des mines, qui lui permettent d'arbitrer, de juger, de conseiller. Ces pratiques sont fondées sur des savoirs de caractère scientifique, par exemple minéralogiques ou géologiques, mais aussi sur des savoirs administratifs, relatifs aux données réglementaires et statistiques, ainsi que sur des savoirs de type juridique, relatifs à la jurisprudence des mines, au droit de propriété et au droit administratif.

En soulignant cet intérêt des pratiques cognitives mobilisées par l'administration des mines, mais aussi par l'ensemble des acteurs concernées par l'activité minière, je me rapproche de ce qui constitue pour moi l'une des thématiques les plus intéressantes en rapport à l'exploitation de ces archives, à savoir l'étude des formes idéelles et matérielles du travail administratif. J'ai analysé pour ma part, ce qui constitue au XIXe siècle le socle intellectuel de l'habitus technocratique en matière administrative, en étudiant la formation reçue par les futurs ingénieurs d'État depuis la préparation à l'École polytechnique jusqu'à leur entrée dans les corps. J'y ai défendu l'idée qu'il existe une sorte d'homologie structurale entre l'organisation du curriculum polytechnicien et l'organisation de la bureaucratie technique en France à cette époque. ॥ reste justement à étudier de manière beaucoup plus systématique et plus fine l'organisation de cette activité technocratique, ses formalités et ses modalités ${ }^{5}$.

C'est là où l'on revient, comme par une boucle, à notre point de départ, c'est-à-dire à la fabrication de l'archive minière elle-même. Celle-ci est le résultat d'une activité qui intéresse l'historien en tant qu'élément dans le processus qui conduit à la prise de décision administrative. Une analyse et une reconstitution, aussi précises que possible, du geste administratif, dans son contexte institutionnel, intellectuel et juridique, fourniraient une contribution tout à fait majeure à une histoire générale des pratiques d'État et des habitus de ses agents. De manière plus précise, il conviendrait de définir et de classer très exactement les modes d'intervention et les formes d'inscription relatives à l'évaluation et à la décision administrative. C'est justement ce que Lionel Latty a entrepris de manière systématique, en étudiant le fonctionnement du Conseil général des mines. Eł c'est à lui, pour terminer cette introduction que je voudrais rendre hommage ici. Car sans cette entreprise courageuse engagée et poursuivie contre vents et marées, sans ces dépouillements marathoniens dont il a bien voulu offrir généreusement les fruits à tous, je crois pouvoir dire que la journée d'études du 15 décembre 2005, dont on trouvera ici les actes, n'aurait sans doute jamais eu lieu.

5 Voir pour la période 1794-1810, I. Laboulais, « Serving science and the state : mining science in France, 1794-1810 ", Minerva, 2008, tome 46, pp. 17-36. Isabelle Laboulais prépare un travail de plus grande ampleur sur les sciences des mines et l'administration sous la Révolution et l'Empire. On pourra se reporter également au dossier d'habilitation de Nathalie Montel « Pour une histoire concrète des savoirs d'État au XIXe siècle : chantiers, vestiges et pratiques ", Université Paris 1 Panthéon Sorbonne, 2008, qui porte sur l'administration des ponts et chaussées. 